\title{
Desarrollo larvario de algunas especies del género Bregmaceros (Pisces: Bregmacerotidae) del sureste del Golfo de México
}

\author{
Jorge Blas-Cabrera ${ }^{1}$, Marina Sánchez-Ramírez ${ }^{1 *}$ \& Alberto Ocaña-Luna ${ }^{1}$ \\ 1 Laboratorio de Ecología, Departamento de Zoología, Escuela Nacional de Ciencias Biológicas del Instituto \\ Politécnico Nacional. Prolongación de Carpio y Plan de Ayala, Col. Casco de Santo Tomas, C.P. 11340, México, D.F. \\ FAX 53963503 \\ * Correspondencia: msanchez@encb.ipn.mx
}

Recibido 07-VIII-2003. Corregido 05-II-2004. Aceptado 29-X-2004.

\begin{abstract}
Larval development of some Bregmaceros species (Pisces: Bregmacerotidae) from the southeast Gulf of Mexico. We redescribe the larval development of Bregmaceros cantori based on 925 specimens ranging from 1.1 to $20.2 \mathrm{~mm}$ in standard length (SL), and describe the larvae of B. atlanticus (27 larvae, 1.7-7.5 mm), B. houdei (six, 1.5-1.9 mm) and B. macclellandi (three, 2.4, 3.4 and $5.4 \mathrm{~mm}$ ). All specimens were collected in the southern Gulf of Mexico from November 27 through December 6, 1998. Larvae were identified to species, and descriptions were made based on pigmentation, and morphometric and meristic characteristics. We defined five development stages: preflexion, flexion, postflexion, transformation and juvenile. In the preflexion stage $B$. cantori displayed a greater growth in mouth size and head length relative to SL (positive allometry); there was negative allometry from the flexion to juvenile stage. B. cantori have the shortest body height and head length, thus being the thinnest; whereas $B$. macclellandi larvae are the most robust ones. From the four species reported from the southern Gulf of Mexico, B. atlanticus larvae are the most pigmented in both head and body, with an homogeneous pattern; $B$. macclellandi presents a different pigmentation pattern consisting in large melanophore groups with a body arrangement that changes from one stage to the next; additionally, from the preflexion stage it develops both the occipital radius and pelvic fins. B. houdei larvae measuring 1.5 to $1.9 \mathrm{~mm}$ have melanophores at the tip of the lower jaw and the head, as well as at the pectoral fin base. Larval development was more pronounced in this B. cantori and B. atlanticus than in specimens from higher latitudes. Rev. Biol. Trop. 54(2): 561-575. Epub 2006 Jun 01.
\end{abstract}

Key words: bregmacerotidae, Bregmaceros, fish larvae, larval development, Gulf of Mexico.

Uno de los principales problemas en los estudios de ictioplancton es la correcta identificación de los organismos, debido a que no siempre se cuenta con la descripción del desarrollo larvario. Ditty (1989) además ha observado que estas descripciones pueden estar incompletas. Por su parte Sánchez-Ramírez y Flores-Coto (1993) encontraron diferencias entre las larvas de carángidos de latitudes mayores en relación con las recolectadas en el sur del Golfo de México.

En el sur del Golfo de México existen pocos trabajos sobre la descripción de las fases larvarias de los peces, dentro de estos se encuentran el de Abundio-López (1987) con algunas especies del orden Pleuronectiformes, de Ordóñez-López y Flores-Coto (1989) sobre las familias Gonostomatidae, Sternoptychidae y Myctophidae, de Sánchez-Iturbe (1993) sobre Syacium gunteri (Bothidae) y por SánchezRamírez y Flores-Coto (1993) y Flores-Coto et al. (1998) con algunas especies de carángidos.

De las especies de la familia Bregmacerotidae que ocurren en el sur del Golfo de México, no se tienen descripciones de su desarrollo ontogénico con organismos 
de estas latitudes, a pesar de que esta familia de acuerdo con Zavala-García y Flores-Coto (1994) está dentro de las más abundantes en el área de estudio, la cual está representada por el género: Bregmaceros, del cual se conocen aproximadamente 12 especies (Stevens y Moser 1996). En el Golfo de México se distribuyen cuatro de ellas: $B$. atlanticus Goode \& Bean, 1886, B. macclellandi Thompson, 1840, B. houdei Saksena \& Richards, 1986 y B. cantori Milliken \& Houde, 1984, las larvas de esta última son las más abundantes en el sur del Golfo de México (Zavala-García y Flores-Coto 1994).

Dentro de las investigaciones realizadas sobre el desarrollo larvario de las especies del género Bregmaceros se encuentran las de Clancey (1956) sobre B. atlanticus de la corriente de Florida, la de D'Ancona y Cavinato (1965) sobre siete especies de este género, de Houde (1981) quien realiza un estudio general sobre $B$. atlanticus y B. macclellandi, así como Houde (1984) con seis especies abarcando tres del Pacífico y tres del Golfo de México. Por otra parte, Milliken y Houde (1984) y Saksena y Richards (1986) describen por primera vez a B. cantori y B. houdei respectivamente, así como su desarrollo larvario.

Estos trabajos corresponden a latitudes mayores por lo que en esta investigación se redescribe el desarrollo larvario de algunas especies de Bregmaceros que ocurren al sur del Golfo de México lo que permitirá una certeza en la identificación de los ejemplares de estas latitudes.

\section{MATERIALES Y MÉTODOS}

El material estudiado proviene del sur del Golfo de México, delimitada por las coordenadas $18^{\circ} 17^{\prime}$ y los $20^{\circ} 59^{\prime} \mathrm{N}$ y los $91^{\circ} 15^{\prime}$ y los $94^{\circ} 50^{\prime} \mathrm{W}$, abarcando la zona oceánica y nerítica de Veracruz, Tabasco y Campeche. La toma de muestras fue realizada a bordo del Buque Oceanográfico "Justo Sierra" de la Universidad Nacional Autónoma de México, del 27 de noviembre al seis de diciembre de 1998.
El material biológico se obtuvo siguiendo la metodología propuesta por Smith y Richardson (1979), los organismos pertenecientes a la familia Bregmacerotidae fueron separados y conservados en alcohol al 70\%, las larvas se identificaron a nivel específico basándose en patrones de pigmentación, características morfométricas y merísticas utilizando literatura especializada: Clancey (1956), D’Ancona y Cavinato (1965), Houde (1981, 1984), Milliken y Houde (1984) y Saksena y Richards (1986). Los ejemplares identificados forman parte de la colección de larvas de peces del laboratorio de Ecología de la Escuela Nacional de Ciencias Biológicas del Instituto Politécnico Nacional bajo el acrónimo ENCBECOL.

Los ejemplares de cada una de las cuatro especies de Bregmaceros se agruparon en las siguientes etapas: preflexión, flexión, postflexión, transformación y juvenil siguiendo el criterio propuesto por Kendall et al. (1984).

Al total de larvas capturadas del género Bregmaceros se les tomaron las siguientes medidas: longitud notocordal (LN) o longitud patrón (LP), longitud de la mandíbula superior (LMS) y longitud de la cabeza (LC) con una precisión de $0.1 \mathrm{~mm}$.

Para las larvas de la especie más abundante, B. cantori, se analizaron las variaciones del tamaño relativo de la boca, de acuerdo al modelo de Shirota (1970) y de la longitud de la cabeza, ambas en relación con la longitud patrón a partir de la ecuación alométrica:

$$
\mathrm{Y}=\mathrm{a}(\mathrm{X})^{\mathrm{b}}
$$

$\mathrm{Y}=$ variable dependiente (tamaño de la boca y longitud de la cabeza).

$\mathrm{X}=$ variable independiente (longitud patrón).

a y $\mathrm{b}=$ son parámetros de la proporción alométrica entre las variables.

Se elaboró para B. cantori, la especie mejor representada tanto en número como en talla, una serie de la menor a la mayor longitud registrada; mientras que para $B$. atlanticus, $B$. macclellandi y $B$. houdei se realizó la descripción de todos los organismos recolectados. A 
los cuales se les tomó, además de las medidas antes mencionadas, altura del cuerpo (AC), longitud hocico-ano (LHA) y diámetro del ojo (DO) que se expresan, al igual que la longitud de la cabeza (LC), como porcentajes promedio en relación con la longitud patrón y el diámetro del ojo en relación con la longitud de la cabeza.

Para la elaboración de los esquemas de las larvas de Bregmaceros se utilizó una cámara clara adaptada a un microscopio estereoscópico.

\section{RESULTADOS}

Se extrajeron un total de 961 organismos pertenecientes a la familia Bregmacerotidae, de los cuales 925 correspondieron a B. cantori en las siguientes etapas de desarrollo: 791 organismos en preflexión (1.1-2.7 mm), 73 en flexión (2.8-4.7 mm), 36 en postflexión (4.8$9.6 \mathrm{~mm})$, seis en transformación (9.7-10.4 mm) y 19 juveniles (10.5-20.2 mm). Mientras que la serie estuvo conformada por 31 organismos: siete en preflexión, cinco en flexión, ocho en postflexión, tres en transformación y ocho juveniles, los cuales presentaban óptimas condiciones de conservación.

De $B$. atlanticus se recolectaron 27 larvas en tres diferentes etapas de desarrollo: 21 en preflexión (1.7-2.8 $\mathrm{mm}$ ), una en flexión $(4.5 \mathrm{~mm})$ y cinco en postflexión $(5.5-7.5 \mathrm{~mm})$; mientras que seis larvas de $B$. houdei fueron identificadas entre 1.5 y $1.9 \mathrm{~mm}$, todas en etapa de preflexión; finalmente de $B$. macclellandi solo tres larvas fueron recolectadas $(2.4 \mathrm{~mm}$ en etapa de preflexión, $3.4 \mathrm{~mm}$ en flexión y 5.4 en postflexión). Estos organismos son los que se utilizaron para la descripción, a pesar de su baja presencia.

\section{Bregmaceros cantori}

Etapa de preflexión (1.1-2.7 mm de LN). Los organismos de $B$. cantori de $1.1 \mathrm{a} 2.0 \mathrm{~mm}$ $(\mathrm{n}=54)$ por lo general presentan de tres a once pequeños melanóforos $(\operatorname{moda}=6-7)$ sobre la superficie ventral del individuo a la altura del estómago; mientras que en ejemplares de 2.1$2.5 \mathrm{~mm}(\mathrm{n}=23)$ se observan de uno a seis melanóforos $($ moda $=1)($ Figs. 1A-1E) y claramente las larvas mayores, 2.6-2.7 mm ( $\mathrm{n}=28)$ tan sólo cuentan con algún vestigio de esta pigmentación, la cual va decreciendo hasta desaparecer conforme la talla del organismo aumenta.

Otro tipo de pigmentación es el que se observó en ejemplares de 1.1 a $2.3 \mathrm{~mm}$, en el que pueden tener de uno a cuatro pequeños melanóforos internos en la línea media ventral del cuerpo (Figs. 1A-1D). También a partir de los $1.3 \mathrm{~mm}$ las larvas ya muestran un melanóforo en la articulación de la mandíbula (Fig. 1B) y a los $1.9 \mathrm{~mm}$ generalmente se les observa pigmento interno cerca de la cápsula ótica.

Las proporciones de la altura del cuerpo, longitud hocico-ano y longitud de la cabeza en esta etapa son $29.2,43.2$ y $27.2 \%$ respectivamente, mientras que el diámetro del ojo es del $44 \%$ (Cuadro 1). A lo largo del desarrollo los organismos se vuelven más delgados, por lo que estas proporciones tienden a disminuir en las siguientes etapas. Esto mismo se observó en la relación alométrica entre la longitud de la cabeza y el tamaño de la boca, donde estos presentaron un mayor desarrollo con relación a la longitud patrón (alometría positiva) (Cuadro 2). Por otra parte, el primordio de las aletas pélvicas aparece entre los 2.5 y $2.6 \mathrm{~mm}$ (Fig. $1 \mathrm{E})$, mientras que el número de miómeros está entre 45 y 48 (Cuadro 3).

Etapa de flexión (2.8-4.7 $\mathrm{mm}$ de $\mathrm{LN})$. Ejemplares que miden más de $2.8 \mathrm{~mm}$ carecen de pigmentación sobre la superficie ventral a la altura del estómago (Fig. 1F). Es posible observar en algunas larvas de $4.1 \mathrm{~mm}$ algunos cúmulos de melanóforos internos por arriba de la línea media lateral en la región posterior del cuerpo, no obstante en la mayoría (70\%) de los especímenes revisados en ésta etapa prácticamente no presentaron este tipo de pigmentación.

Por otra parte, a los $2.8 \mathrm{~mm}$ el urostilo está flexionado y a los $2.9 \mathrm{~mm}$ se inicia la formación de los radios de la aleta caudal, en estas tallas inicia el desarrollo de las aletas dorsal y anal, observándose sus primeros radios entre 


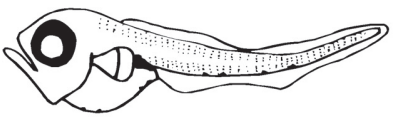

A

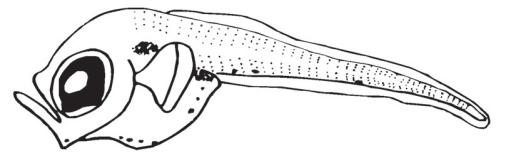

C

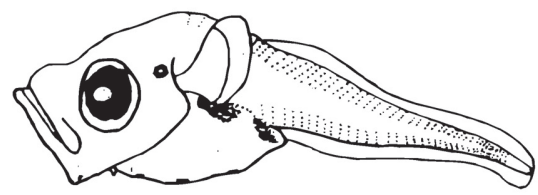

E

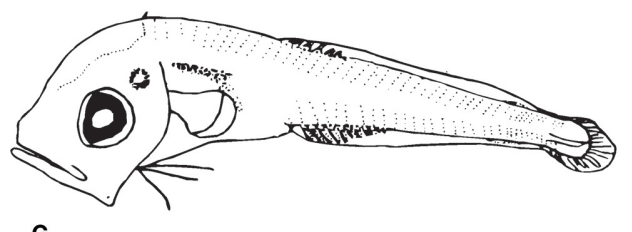

G
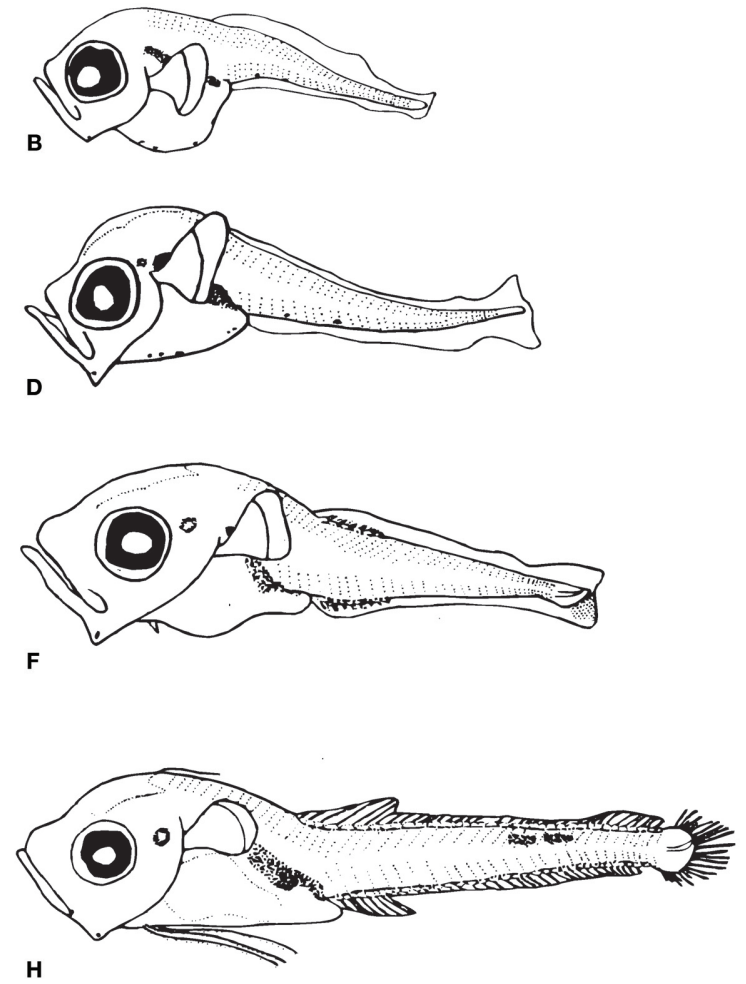

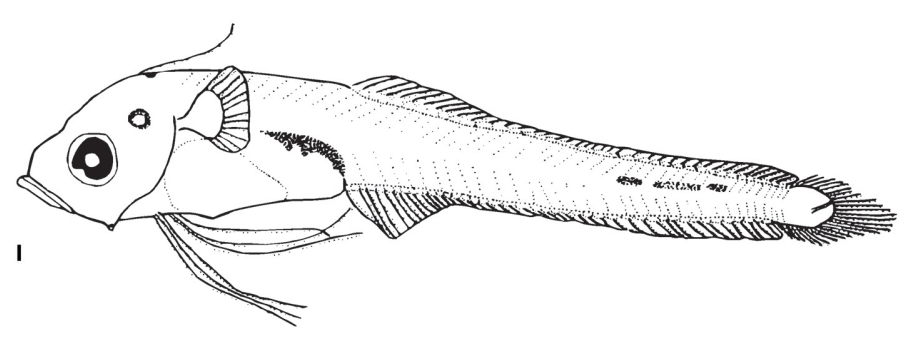

Fig. 1. Larvas de Bregmaceros cantori en etapa de preflexión: A. $1.2 \mathrm{~mm}$, B. $1.3 \mathrm{~mm}$, C. $1.4 \mathrm{~mm}$, D. $2.1 \mathrm{~mm}$ y E. $2.5 \mathrm{~mm}$; flexión: F. 3.0 mm y G. 4.0 mm; posflexión: H. 5.5 mm y I. $9.2 \mathrm{~mm}$.

Fig. 1. Larvae of Bregmaceros cantori in preflexion stage: A. $1.2 \mathrm{~mm}$, B. $1.3 \mathrm{~mm}$, C. $1.4 \mathrm{~mm}$, D. $2.1 \mathrm{~mm}$ and E. $2.5 \mathrm{~mm}$; flexion stage: F. $3.0 \mathrm{~mm}$ and G. $4.0 \mathrm{~mm}$; postflexion stage: H. $5.5 \mathrm{~mm}$ y I. $9.2 \mathrm{~mm}$.

los 2.9 y $3.0 \mathrm{~mm}$, mientras que el radio occipital aparece a partir de los $4.0 \mathrm{~mm}$, en esta talla las aletas pélvicas prácticamente alcanzan la longitud de la aleta pectoral (Fig. 1G).

La altura del cuerpo respecto a la longitud notocordal es menor que en la etapa anterior (27.5\%), de igual manera el diámetro del ojo en relación con la longitud de la cabeza también disminuyó (31.1\%), mientras que la longitud hocico-ano y de la cabeza respecto a la longitud notocordal aumentaron ligeramente en comparación con preflexión (45.6 y 29.1\% respectivamente) (Cuadro 1), sin embargo al analizar el total de larvas en esta etapa, se observó un crecimiento alométrico negativo tanto del tamaño de la cabeza como de la boca con relación a la longitud notocordal (Cuadro 2).

Etapa de postflexión (4.8-9.6 mm de LP). A partir de esta etapa es evidente y constante la 
CUADRO 1

Proporciones del cuerpo de las especies de Bregmaceros en las diferentes etapas de desarrollo

TABLE 1

Body proportions of the species of Bregmaceros in the different development stages

\begin{tabular}{|c|c|c|c|c|c|}
\hline & & $\begin{array}{c}\text { B. cantori } \\
(\%)\end{array}$ & $\begin{array}{c}\text { B. atlanticus } \\
(\%)\end{array}$ & $\begin{array}{c}\text { B. macclellandi } \\
(\%)\end{array}$ & $\begin{array}{c}\text { B. houdei } \\
(\%)\end{array}$ \\
\hline & Preflexión & 29.2 & 32.5 & 41.9 & 30.1 \\
\hline & Flexión & 27.5 & 28.0 & 41.9 & - \\
\hline \multirow[t]{5}{*}{$\mathrm{AC}$} & Postflexión & 19.5 & 25.2 & 31 & - \\
\hline & Transformación & 13.8 & - & - & - \\
\hline & Juvenil & 13.7 & - & - & - \\
\hline & Preflexión & 43.2 & 50.3 & 50.6 & 43.32 \\
\hline & Flexión & 45.6 & 52.8 & 47.5 & - \\
\hline \multirow[t]{5}{*}{ LHA } & Postflexión & 40.3 & 45.8 & 47.6 & - \\
\hline & Transformación & 37.6 & - & - & - \\
\hline & Juvenil & 37.5 & - & - & - \\
\hline & Preflexión & 44.0 & 45.7 & 36.7 & 48.0 \\
\hline & Flexión & 31.1 & 31.2 & 31.4 & - \\
\hline \multirow[t]{5}{*}{ DO } & Postflexión & 24.8 & 26.2 & 30 & - \\
\hline & Transformación & 19.5 & - & - & - \\
\hline & Juvenil & 21.9 & - & - & - \\
\hline & Preflexión & 27.2 & 28.8 & 37.5 & 26.8 \\
\hline & Flexión & 29.1 & 28.9 & 32.4 & - \\
\hline \multirow[t]{3}{*}{$\mathrm{LC}$} & Postflexión & 23.1 & 27.9 & 25.9 & - \\
\hline & Transformación & 20.2 & - & - & - \\
\hline & Juvenil & 18.9 & - & - & - \\
\hline
\end{tabular}

$\mathrm{AC}=$ altura del cuerpo. $\mathrm{LHA}=$ longitud hocico-ano. $\mathrm{DO}=$ diámetro del ojo. $\mathrm{LC}=$ longitud de la cabeza. Nota: - ausencia de organismos.

presencia de los melanóforos internos en la región posterior del cuerpo que habían aparecido en algunos ejemplares de la etapa de flexión (Fig. 1H). En organismos mayores a $6.5 \mathrm{~mm}$ se pueden presentar uno o más melanóforos sobre la cabeza, justo detrás de donde inicia el radio occipital (Fig. 1I).

En esta etapa hay una mayor formación de los radios de las aletas anal, dorsal, caudal y pectorales. A los $5.5 \mathrm{~mm}$ las aletas pélvicas sobrepasan la longitud de la aleta pectoral y a los $9.2 \mathrm{~mm}$ las pélvicas alcanzan el inicio de la aleta anal mientras que el radio occipital llega al borde de la aleta pectoral. La altura del cuerpo es menor en comparación con las etapas de preflexión y flexión (19.5\%), también hay una disminución en la proporción del diámetro del ojo (24.8\%), de la longitud hocico-ano (40.3\%) y de la longitud de la cabeza (23.1\%). A partir de la etapa anterior hasta la etapa de juvenil hay 
CUADRO 2

Parámetros de la relación alométrica de Bregmaceros cantori en sus etapas de desarrollo

TABLE 2

Parameters of the allometry ratios of Bregmaceros cantori in its development stages

\begin{tabular}{lccccc} 
& \multicolumn{5}{c}{ Preflexión $(\mathrm{n}=789)$} \\
& $\mathrm{a}$ & $\mathrm{b}$ & $\mathrm{R}^{2}$ & alometría & $\mathrm{T}_{\mathrm{b}}$ \\
Tamaño de la boca & 0.187 & 1.376 & 79.527 & + & 15.113 \\
Longitud de la cabeza & 0.213 & 1.305 & 83.346 & + & 14.673
\end{tabular}

Flexión $(\mathrm{n}=72)$

$\begin{array}{lccccc} & \mathrm{a} & \mathrm{b} & \mathrm{R}^{2} & \text { alometría } & \mathrm{T}_{\mathrm{b}} \\ \text { Tamaño de la boca } & 0.349 & 0.711 & 53.729 & - & -3.660 \\ \text { Longitud de la cabeza } & 0.331 & 0.881 & 60.789 & - & -1.413\end{array}$

\begin{tabular}{lccccc} 
& \multicolumn{5}{c}{ Postflexión $(\mathrm{n}=36)$} \\
& $\mathrm{a}$ & $\mathrm{b}$ & $\mathrm{R}^{2}$ & alometría & $\mathrm{T}_{\mathrm{b}}$ \\
Tamaño de la boca & 0.488 & 0.466 & 81.17 & - & -13.854 \\
Longitud de la cabeza & 0.426 & 0.666 & 92.25 & - & -10.099
\end{tabular}

\begin{tabular}{lccccc} 
& \multicolumn{5}{c}{ Transformación-Juvenil $(\mathrm{n}=25)$} \\
& $\mathrm{a}$ & $\mathrm{b}$ & $\mathrm{R}^{2}$ & alometría & $\mathrm{T}_{\mathrm{b}}$ \\
Tamaño de la boca & 0.284 & 0.671 & 89.29 & - & -6.792 \\
Longitud de la cabeza & 0.284 & 0.852 & 94.94 & - & -3.599
\end{tabular}

$\mathrm{R}^{2}=$ valor de correlación. $\mathrm{a}=$ ordenada al origen. $\mathrm{b}=$ pendiente. $\mathrm{T}_{\mathrm{b}}=\mathrm{t}$ de la $\mathrm{b} . \mathrm{n}=$ número de organismos analizados.

un crecimiento menor del tamaño de la cabeza y de la boca con relación a la longitud patrón (alometría negativa) (Cuadro 2).

Transformación (9.7-10.4 mm de LP). La pigmentación es más evidente en este estadio que en el anterior, ya que se registró un incremento de melanóforos internos que se van esparciendo dorsalmente desde la región caudal hacia la parte media del cuerpo, también hay aparición de algunos pequeños pigmentos externos hacia la parte caudal (Figs. 2A y 2B).

En esta etapa se registra un cambio en los organismos de larva a juvenil, en la cual la cabeza y el cuerpo van adquiriendo la forma del adulto, donde prácticamente ya se ha completado la formación de los radios de las aletas dorsal (44-48), anal (47-48), caudal (30-31) y pectorales (15-17) (Cuadro 3). Conforme se han desarrollado los organismos, estos presentan un cuerpo más alargado lo cual se sigue reflejando en una disminución de las proporciones entre la altura del cuerpo (13.8\%), longitud hocico-ano (37.6\%) y longitud de la cabeza $(20.2 \%)$, mientras que la proporción del diámetro del ojo es del $19.5 \%$.

Juvenil (10.5-20.2 mm de LP). En este estadio de desarrollo, $B$. cantori tiene un incremento de melanóforos internos dorsales a lo largo de la columna vertebral, desde el pedúnculo caudal hacia la altura del inicio de la aleta pectoral, también presentan algunos pigmentos externos dorsales en la región posterior (Fig. 2C). 
CUADRO 3

Características morfométricas y merísticas de larvas de Bregmaceros cantori, sur del Golfo de México

TABLE 3

Morphometric and meristic characteristics of the larvae of Bregmaceros cantori, southern Gulf of Mexico

$\begin{array}{cccccccccc}\begin{array}{c}\text { LN/LP } \\ (\mathrm{mm})\end{array} & \begin{array}{c}\text { Altura } \\ \text { Cuerpo } \\ (\mathrm{mm})\end{array} & \begin{array}{c}\text { Longitud } \\ \text { Cabeza } \\ (\mathrm{mm})\end{array} & \begin{array}{c}\text { Diámetro } \\ \text { Ojo } \\ (\mathrm{mm})\end{array} & \begin{array}{c}\text { Longitud } \\ \text { Hocico-ano } \\ (\mathrm{mm})\end{array} & \begin{array}{c}\text { Aleta } \\ \text { Dorsal }\end{array} & \begin{array}{c}\text { Aleta } \\ \text { Anal }\end{array} & \begin{array}{c}\text { Aleta } \\ \text { Pectoral }\end{array} & \begin{array}{c}\text { Aleta } \\ \text { Caudal } \\ \text { (radios totales) }\end{array} & \text { MIÓMEROS } \\ 1.1 & 0.27 & 0.30 & 0.12 & 0.45 & - & - & - & - & - \\ 1.2 & 0.38 & 0.30 & 0.17 & 0.53 & - & - & - & - & 47 \\ 1.3 & 0.39 & 0.30 & 0.15 & 0.56 & - & - & - & - & 45 \\ 1.4 & 0.39 & 0.40 & 0.17 & 0.56 & - & - & - & - & 46 \\ 1.5 & 0.45 & 0.50 & 0.20 & 0.66 & - & - & - & - & 46 \\ 2.0 & 0.62 & 0.50 & 0.21 & 0.90 & - & - & - & - & 47 \\ 2.5 & 0.75 & 0.70 & 0.29 & 1.16 & - & - & - & - & 48\end{array}$

Flexión

$\begin{array}{llllllllll}2.8 & 0.90 & 0.90 & 0.30 & 1.48 & * & 6 & - & * & 47 \\ 3.0 & 0.87 & 0.80 & 0.30 & 1.43 & 6 & 8 & - & 9 & 45 \\ 3.5 & 1.01 & 1.10 & 0.30 & 1.56 & 7 & 10 & - & 11 & 48 \\ 4.0 & 0.95 & 1.10 & 0.33 & 1.63 & 9 & 12 & - & * & 47 \\ 4.3 & 1.04 & 1.20 & 0.33 & 1.82 & 10 & 13 & - & 21 & 47\end{array}$

$\begin{array}{llllllllll}4.9 & 1.16 & 1.20 & 0.33 & 2.23 & 31 & 35 & 3 & 21 & 48 \\ 5.1 & 1.17 & 1.30 & 0.35 & 2.18 & * & 34 & * & 21 & 47 \\ 5.5 & 1.20 & 1.40 & 0.38 & 2.54 & * & 36 & 7 & 23 & 48 \\ 6.1 & 1.20 & 1.40 & 0.38 & 2.45 & 36 & 41 & 6 & 24 & 48 \\ 6.8 & 1.29 & 1.50 & 0.38 & 2.67 & 38 & 41 & 11 & 23 & 48 \\ 7.5 & 1.43 & 1.70 & 0.39 & 2.76 & 37 & 41 & 14 & 25 & 47 \\ 8.6 & 1.29 & 1.70 & 0.39 & 3.10 & 37 & 45 & 15 & 28 & 46 \\ 9.5 & 1.46 & 2.10 & 0.42 & 3.44 & 39 & 43 & 14 & 29 & 46\end{array}$

Transformación

$\begin{array}{cccccccccc}9.7 & 1.32 & 1.90 & 0.41 & 3.72 & 44 & 47 & 15 & 31 & 47 \\ 10.0 & 1.40 & 2.00 & 0.39 & 3.80 & 48 & 48 & 15 & 30 & 47 \\ 10.4 & 1.43 & 2.20 & 0.39 & 3.80 & 47 & 48 & 16 & 31 & 47\end{array}$

Juvenil

$\begin{array}{llllllllll}11.0 & 1.52 & 2.20 & 0.44 & 4.30 & 46 & 47 & 17 & 31 & 47 \\ 11.7 & 1.60 & 2.30 & 0.50 & 4.55 & 45 & 47 & 16 & 29 & 45 \\ 12.3 & 1.60 & 2.40 & 0.48 & 4.50 & 46 & 49 & 16 & 30 & 46 \\ 14.0 & 1.82 & 2.70 & 0.53 & 5.15 & 46 & 47 & 17 & 31 & 47 \\ 15.0 & 2.13 & 2.80 & 0.63 & 5.58 & 47 & 47 & 18 & 32 & 47 \\ 15.8 & 2.18 & 2.90 & 0.69 & 5.95 & 45 & 48 & 16 & 31 & 46 \\ 19.6 & 2.54 & 3.40 & 0.78 & 6.96 & 45 & 48 & 18 & 32 & 47 \\ 20.2 & 3.04 & 3.70 & 0.95 & 7.76 & 46 & 48 & 16 & 31 & 48\end{array}$

Nota: * No fue posible realizar el conteo de miómeros o radios. 

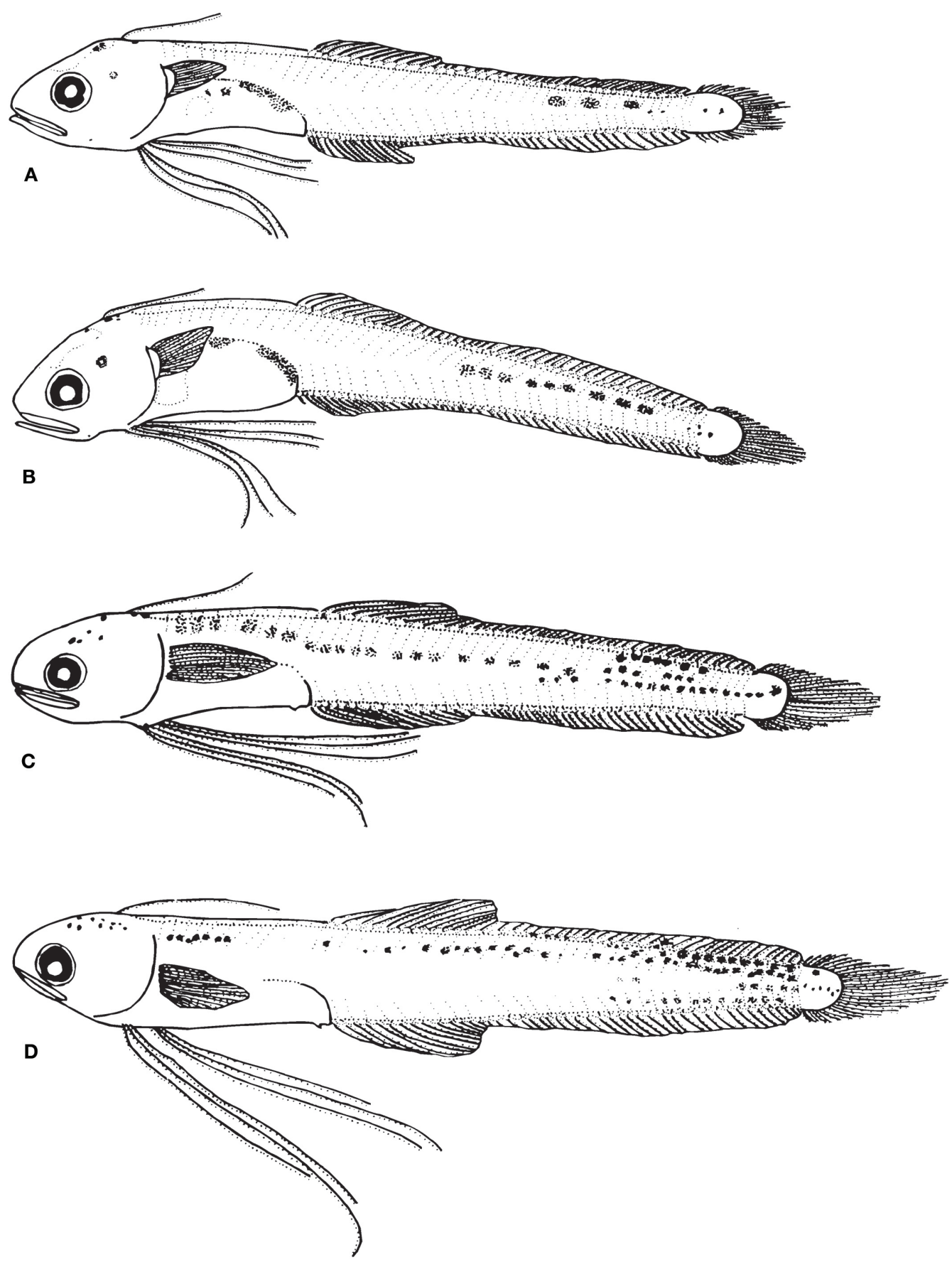

Fig. 2. Larvas de Bregmaceros cantori en etapa de transformación: A. 9.7 mm y B. 10.0 mm; etapa juvenil: C. 12.3 mm y D. $19.6 \mathrm{~mm}$.

Fig. 2. Larvae of Bregmaceros cantori in transformation stage: A. $9.7 \mathrm{~mm}$ and B. $10.0 \mathrm{~mm}$; juvenile stage: C. $12.3 \mathrm{~mm}$ and D. $19.6 \mathrm{~mm}$. 
En tallas mayores hay un aumento de melanóforos pequeños dorsales externos, que se extienden a lo largo del organismo desde el pedúnculo caudal hacia la cabeza, así como algunos melanóforos ventrales internos en la parte caudal (Fig. 2D).

Desde la etapa de transformación ya no hay mucha variación en la formación de los radios de las aletas, en la cual los organismos ya cuentan con las características de los adultos, presentando entre 45 y 48 radios dorsales, de 47 a 49 radios anales, entre 16 y 18 radios pectorales y de 29 a 32 radios caudales, así como de 45 a 48 miómeros totales (Cuadro 3). La longitud de las aletas pélvicas en esta etapa sobrepasan considerablemente el inicio de la aleta anal y el radio occipital abarca más de la mitad de la longitud hocico-ano.

De igual manera, entre la etapa de transformación y juvenil casi no cambia la altura del cuerpo (13.7\%) ni la longitud hocico-ano $(37.5 \%)$, sin embargo si hay un ligero decremento entre la longitud de la cabeza $(18.9 \%)$ y un incremento en el diámetro del ojo (21.9\%) (Cuadro 1). Por otra parte, cabe resaltar que en esta etapa ya hay formación de escamas en los organismos.

\section{Bregmaceros atlanticus}

La larva más pequeña capturada mide $1.7 \mathrm{~mm}$ mostrando un melanóforo grande en la parte media ventral del cuerpo (Fig. 3A), el cual se incrementa en organismos de 1.8 a $1.9 \mathrm{~mm}$ observándose dos grupos de melanóforos ventrales y uno dorsal. Tallas de 2.0 a $2.8 \mathrm{~mm}$ pueden tener de tres a cuatro grupos de melanóforos ventrales y de dos a tres dorsales en el cuerpo, este tipo de pigmentación se puede observar en forma difusa (Fig. 3B). Todas las larvas $(n=27)$ poseen melanóforos en la cabeza, en las aletas pectorales, en la punta de la mandíbula superior y en el ángulo de la mandíbula, una hilera de pequeños pigmentos en la mandíbula inferior, así como varios melanóforos en la parte ventral del estómago.

Los ejemplares con tallas de 1.7 a $2.8 \mathrm{~mm}$ $(n=21)$ se encontraban en etapa de preflexión, en la cual las larvas presentan una altura del cuerpo, longitud hocico-ano y longitud de la cabeza del $32.5,50.3$ y $28.8 \%$ respectivamente, siendo estas proporciones mayores que B. cantori, mientras que el diámetro del ojo es del 47.5\% (Cuadro 1).

Por otro lado se examinó un organismo en etapa de flexión de $4.5 \mathrm{~mm}$ muy pigmentado, el cual presenta melanóforos en la cabeza, aletas pectorales, cuerpo y estómago, con pequeños melanóforos en la región caudal y otros más esparcidos en las aletas anal y caudal. En esta talla se observa el brote del radio occipital (Fig. 3C).

Por último fueron examinadas cinco larvas entre 5.5 y $7.5 \mathrm{~mm}$, las que se encontraban en etapa de postflexión, con melanóforos en todo el cuerpo: cabeza, estómago y en las aletas pectorales, los cuales son más pequeños hacia la región caudal. También presentan pequeños puntos de pigmentos en la aleta caudal, ventral y dorsal (Figs. 3D y 3E). A los $7.5 \mathrm{~mm}$ las aletas pélvicas sobrepasan ligeramente el inicio de la aleta anal. Por otra parte, las proporciones de la altura del cuerpo, la longitud hocico-ano y longitud de la cabeza siguen siendo mayores que las de $B$. cantori $(25.2,45.8$ y $27.9 \%$ respectivamente), así mismo el diámetro del ojo es del 26.2\% (Cuadro 1).

\section{Bregmaceros houdei}

De esta especie sólo se recolectaron seis organismos entre 1.5 y $1.9 \mathrm{~mm}$ en preflexión, los cuales no presentaron pigmentación en el cuerpo, pero sí algunos melanóforos en la nuca y en la punta de la mandíbula inferior, así como otros pequeños melanóforos en la base de las aletas pectorales, en la parte anterior del cerebro y en la región ventral-anterior del estómago (Figs. 4A y 4B). En cuanto a las proporciones de la altura del cuerpo (30.1\%), longitud hocico-ano $(43.3 \%)$ y longitud de la cabeza $(26.8 \%)$ son muy similares a $B$. cantori, siendo ambas especies de complexión delgada, aunque el diámetro del ojo de $B$. houdei si es ligeramente superior $(48 \%)$.

De las cuatro especies de Bregmaceros registradas en el área de estudio, las larvas de 

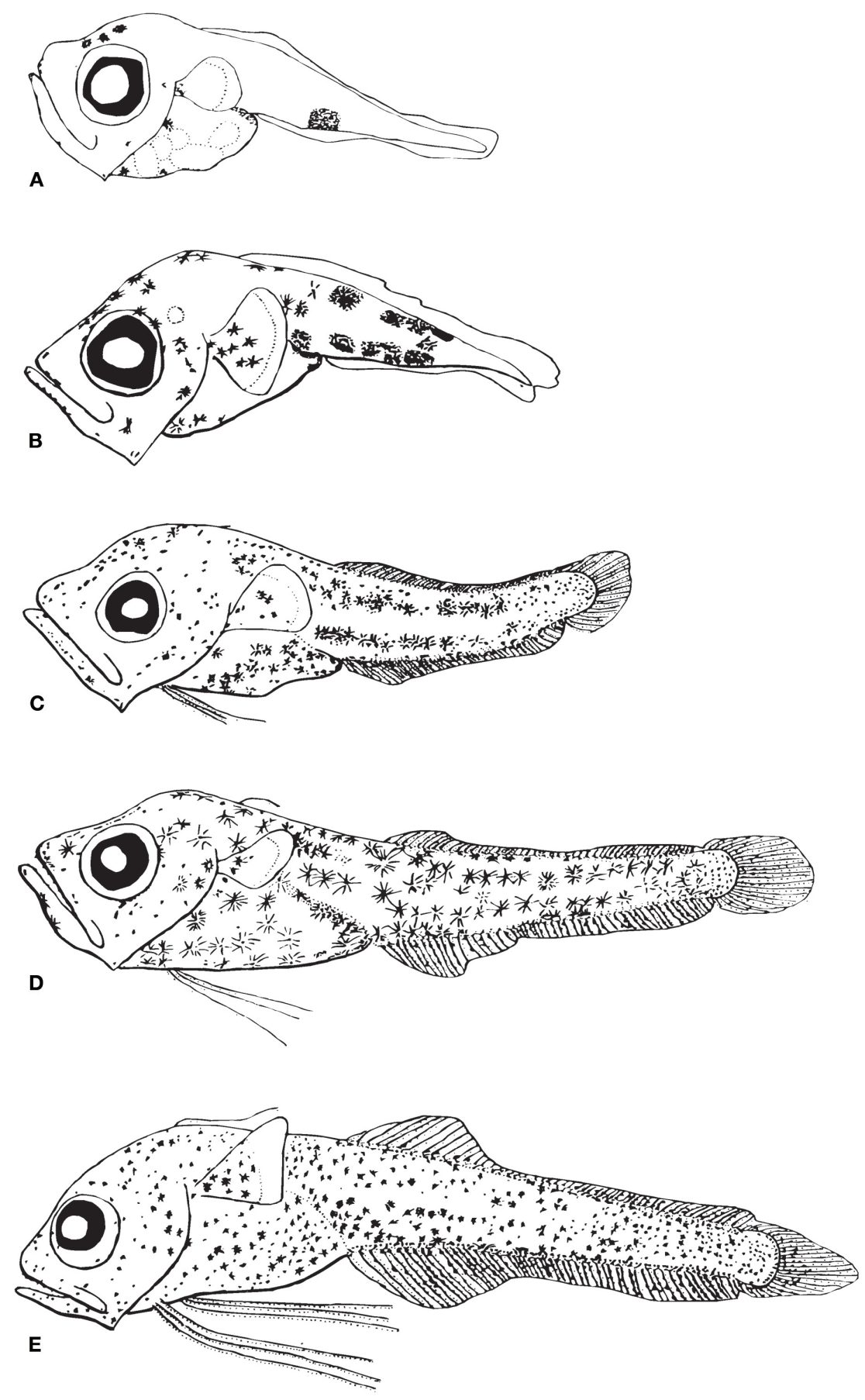

Fig. 3. Larvas de Bregmaceros atlanticus en etapa de preflexión: A. $1.7 \mathrm{~mm}$ y B. $2.4 \mathrm{~mm}$; flexión: C. $4.5 \mathrm{~mm}$; posflexión: D. $5.5 \mathrm{~mm}$ y E. $7.5 \mathrm{~mm}$.

Fig. 3. Larvae of Bregmaceros atlanticus in preflexion stage: A. $1.7 \mathrm{~mm}$ and B. $2.4 \mathrm{~mm}$; flexion stage: C. $4.5 \mathrm{~mm}$; postflexion stage: D. $5.5 \mathrm{~mm}$ and E. $7.5 \mathrm{~mm}$. 

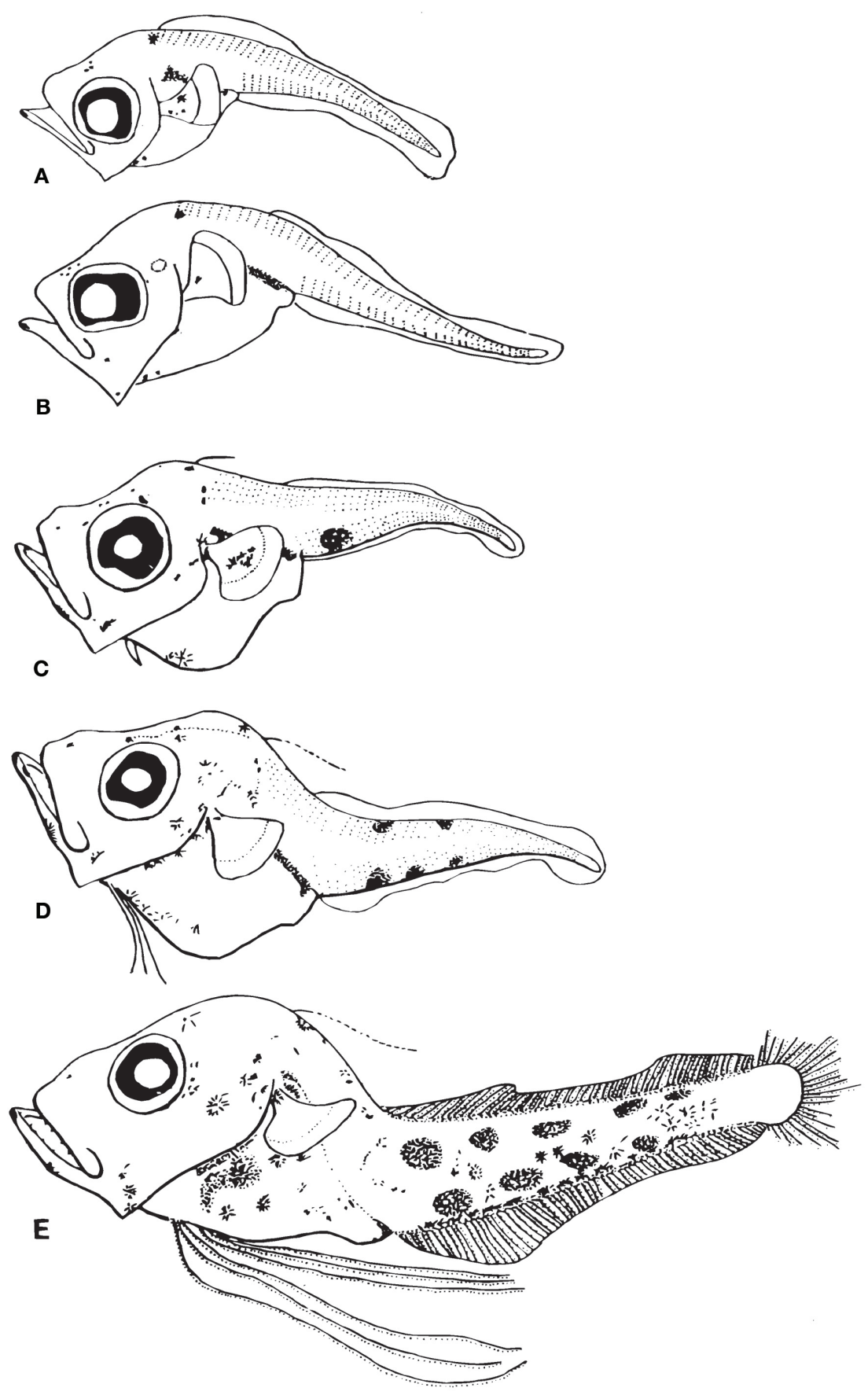

Fig. 4. Larvas de Bregmaceros houdei en etapa de preflexión: A. 1.5 mm y B. $1.9 \mathrm{~mm}$. Larvas de Bregmaceros macclellandi en etapa de preflexión: C. $2.4 \mathrm{~mm}$, flexión: D. $3.4 \mathrm{~mm}$; posflexión: E. $5.4 \mathrm{~mm}$.

Fig. 4. Larvae of Bregmaceros houdei in preflexion stage: A. $1.5 \mathrm{~mm}$ and B. $1.9 \mathrm{~mm}$. Larvae of Bregmaceros macclellandi in preflexion stage: C. $2.4 \mathrm{~mm}$, flexion stage: D. $3.4 \mathrm{~mm}$; postflexion stage: E. $5.4 \mathrm{~mm}$. 
B. cantori y B. houdei se pueden separar por la presencia de pigmentos en la punta de la mandíbula inferior y en la base de las aletas pectorales, de este última, además de presentar un radio occipital desde la flexión como lo menciona Saksena y Richards (1986), mientras que $B$. cantori carece de este patrón de pigmentación y presenta sólo un brote del radio occipital en esta etapa; en las siguientes etapas de desarrollo $B$. cantori ya presenta pigmentación en el cuerpo, con un incremento de melanóforos dorsales a través de todo el tronco, mientras que juveniles de $B$. houdei descritos por Saksena y Richards (1986) tienen un mayor desarrollo de melanóforos dorsales hasta el inicio de la aleta dorsal, así como en la cabeza y estómago.

\section{Bregmaceros macclellandi}

Fueron capturados tres ejemplares, el más pequeño de $2.4 \mathrm{~mm}$ el cual estaba en etapa de preflexión, se le observa un melanóforo grande en la parte ventral del cuerpo después del ano, con pigmento en la punta de la mandíbula inferior y en el ángulo de la mandíbula, así como un melanóforo estrellado en la región ventral de la masa visceral, en las aletas pectorales, sobre la cabeza y algunos a lo largo de la mandíbula inferior. En esta talla ya tienen desarrollados tanto el radio occipital como las aletas pélvicas (Fig. 4C).

El organismo de $3.4 \mathrm{~mm}$ (etapa de flexión) presenta tres grupos de melanóforos ventrales y dos dorsales en el cuerpo, observando que dos pares de melanóforos se ubican a la misma altura. Hacia la región caudal no hay pigmento y al igual que la talla anterior éste espécimen mostró algunos pequeños melanóforos en la parte ventral de la masa visceral, en la base de las aletas pélvicas, en la cabeza, en la punta y a lo largo de la mandíbula inferior (Fig. 4D).

El tercer organismo capturado de $5.4 \mathrm{~mm}$ (etapa de postflexión) se le observa una línea de cuatro grupos de melanóforos ventrales y cinco dorsales, además de tener algunos pequeños pigmentos en la parte posterior del cuerpo, en la línea media ventral así como en la línea media del cuerpo, sin embargo en la región caudal hay ausencia de melanóforos. Por otra parte, el pigmento en la punta de la mandíbula inferior sigue presente, así como en la cabeza, estómago, en la base de las aletas pectorales y aparecen algunos puntos pequeños en la parte posterior de la aleta anal. En esta talla las aletas pélvicas están muy desarrolladas, sobrepasando el inicio de la aleta anal (Fig. 4E).

Esta especie presentó la mayor altura del cuerpo con respecto a las otras tres especies de Bregmaceros registradas, con el $41.9 \%$ tanto en preflexión como en flexión y el $31.0 \%$ en postflexión. Mientras que la longitud hocico-ano es del $50.6 \%$ para preflexión, de $47.5 \%$ en flexión y del $47.6 \%$ para la etapa de postflexión, estas proporciones son similares a $B$. atlanticus pero muy superiores a $B$. cantori. En cambio, el diámetro del ojo es menor en preflexión (36.7\%) que las otras especies de Bregmaceros, sin embargo es similar en flexión (31.4\%), pero el ojo es mayor en postflexión (30\%) que B. cantori y $B$. atlanticus. Por último, la longitud de la cabeza también es más grande que las otras especies con el $37.5 \%$ para preflexión y $32.4 \%$ en flexión (Cuadro 1).

\section{DISCUSIÓN}

Son varias las diferencias observadas en las larvas descritas en este trabajo con las de otras latitudes, sobre todo en las primeras etapas de desarrollo.

Larvas de $B$. cantori de 1.1 a $2.3 \mathrm{~mm}$ presentaron algunos melanóforos internos en la línea media ventral del cuerpo, así como pigmento en el ángulo de la mandíbula a partir de los 1.3 mm, en cambio Houde (1981) con larvas descritas al norte del Golfo de México, observó el pigmento en la línea media ventral del cuerpo en organismos de mayor talla entre los 2.0 y $3.0 \mathrm{~mm}$, mientras que el pigmento en el ángulo de la mandíbula lo refiere desde los $3.0 \mathrm{~mm}$.

Por otra parte esta especie a los $1.9 \mathrm{~mm}$ generalmente ya se observa pigmento interno cerca de la cápsula ótica, sin embargo Houde (1984) lo registra a partir de los $5.0 \mathrm{~mm}$. Otro 
tipo de desfasamiento es el observado en tallas de $2.8 \mathrm{~mm}$, los cuales ya no presentan melanóforos sobre la superficie ventral del estómago, sin embargo Milliken y Houde (1984) mencionan que este tipo de pigmentación desaparece en tallas de $5.0 \mathrm{~mm}$.

Otra diferencia en las larvas de B. cantori es el brote del radio occipital que se observó desde los $4.0 \mathrm{~mm}$, lo cual difiere con lo registrado por Houde (1981) y Milliken y Houde (1984) quienes mencionan que el radio occipital aparece entre los 6.0 y $7.0 \mathrm{~mm}$. Las diferencias sin embargo son menores en organismos más grandes, ya que a los $6.5 \mathrm{~mm}$ de LP pueden aparecer pigmentos en la cabeza que se van incrementando en larvas mayores, esto es similar a lo registrado por Houde (1981) quien menciona que a los $7.0 \mathrm{~mm}$ pueden aparecer de uno a dos melanóforos sobre la cabeza y conforme los organismos se desarrollan el número de melanóforos se incrementa, sin embargo se sigue observando que la pigmentación aparece unos milímetros antes en las larvas analizadas en este trabajo.

$\mathrm{Al}$ analizar este tipo de comparaciones, es evidente observar que a través de las diferentes etapas de desarrollo las larvas de $B$. cantori del sur del Golfo de México presentan un desarrollo más rápido que las registradas al este del Golfo de México (Plataforma de Florida) por Houde (1981) o al oeste del Océano Atlántico por Milliken y Houde (1984).

También se observaron algunas diferencias en larvas de $B$. atlanticus con ejemplares examinados por otros autores, por ejemplo Houde (1984) observó el brote del radio occipital entre los 5.0 y $5.5 \mathrm{~mm}$, mientras que en este trabajo desde los $4.5 \mathrm{~mm}$.

Los ejemplares de esta especie, entre 4.5 y $5.5 \mathrm{~mm}$ presentaron melanóforos en la cabeza, abdomen y cuerpo, con un radio occipital muy corto el cual no sobrepasa la longitud de la aleta pectoral, sin embargo esto difiere del propuesto por Clancey (1956) con un organismo de talla similar $(4.9 \mathrm{~mm})$, en el que hay ausencia de pigmentación en el cuerpo, así como larvas de 3.2 y $5.2 \mathrm{~mm}$ con un radio occipital largo que sobrepasa considerablemente el inicio de la aleta dorsal, con grupos de pigmentos dorsales y ventrales en el cuerpo. Estas diferencias, tanto en pigmentación como en la longitud del radio, sugieren que la descripción elaborada por Clancey (1956) presenta una mezcla de larvas de $B$. atlanticus y $B$. macclellandi.

De las larvas de $B$. houdei que se encontraron entre 1.5 y $1.9 \mathrm{~mm}$, presentaron melanóforos en la base de las aletas pectorales, así como en la punta de la mandíbula inferior, en la cabeza y abdomen, mientras que Houde (1981) y Saksena y Richards (1986) quienes describen a esta especie, no mencionan pigmentación alguna en la base de las aletas pectorales. Por otra parte larvas de B. atlanticus y B. macclellandi también la presentan, sin embargo estas son especies muy pigmentadas.

Fue posible observar un desarrollo más rápido de las diferentes especies de larvas de Bregmaceros del sur del Golfo de México, en relación con las registradas en latitudes mayores a las del área de estudio, sobre todo de $B$. cantori, de la cual se dio seguimiento desde su etapa larvaria hasta la etapa de juvenil. Blaxter (1969) menciona que el tiempo que puede pasar una larva de una etapa a otra hasta convertirse en adulto, puede durar varios días en especies tropicales, hasta semanas o meses en organismos de latitudes mayores. Laurence (1978) observó en Gadus morhua y Melanogrammus aeglefinus (Gadiformes) que estas especies se desarrollan más rápido a mayores temperaturas del agua. De manera similar Houde (1989) y Morse (1989) mencionan que la duración de un estadio en larvas de peces marinos, es mayor en temperaturas bajas o en mayores latitudes. Lo anterior se ha observado en las etapas larvarias de algunos carángidos del sur del Golfo de México, presentando estos ejemplares un desarrollo más rápido en relación con los que habitan en el norte del Golfo de México y en la costa Atlántica de los Estados Unidos (Sánchez-Ramírez y Flores-Coto 1993).

\section{AGRADECIMIENTOS}

Agradecemos a Felipe Vázquez Gutiérrez (ICMyL-UNAM) director del proyecto 
"Evaluación prospectiva para el programa de monitoreo continuo del efecto ambiental de la actividad petrolera en el Golfo de México, monitoreo ambiental para el proyecto de modernización y optimización del campo Cantarel" del cual forma parte la campaña oceanográfica SGM4, así como a Laura Sánchez Velasco (CICIMAR-IPN) por la recolección y facilidades de acceso al material biológico.

\section{RESUMEN}

Se redescribe el desarrollo larvario de Bregmaceros cantori con 925 organismos en tallas de 1.1 a $20.2 \mathrm{~mm}$ de longitud patrón (LP) y se describen algunos ejemplares de $B$. atlanticus (27 larvas de 1.7 a $7.5 \mathrm{~mm}$ ), de $B$. houdei (seis organismos de 1.5 a $1.9 \mathrm{~mm}$ ) y de $B$. macclellandi (tres larvas de 2.4, 3.4 y $5.4 \mathrm{~mm}$ ) del sur del Golfo de México (27 noviembre - 6 diciembre 1998). Las larvas se identificaron a nivel específico y se describieron con base en pigmentación, y características morfométricas y merísticas. En la etapa de preflexión B. cantori presentó un mayor crecimiento del tamaño de la boca y de la longitud de la cabeza en relación con la longitud patrón (alometría positiva); en cambio de la etapa de flexión a la etapa de juvenil decrecen (alometría negativa). B. cantori es la especie con la menor altura del cuerpo y longitud de la cabeza, siendo así la más delgada. Las larvas de $B$. macclellandi son las más robustas. De las cuatro especies que se reportan en el sur del Golfo de México, las larvas de B. atlanticus son las que tienen la mayor pigmentación tanto en la cabeza como en el cuerpo, de manera uniforme; los ejemplares de $B$. macclellandi muestran un patrón de pigmentación diferente: grandes grupos de melanóforos cuya ubicación cambia con la etapa; además ya en la etapa de preflexión tiene desarrollo del radio occipital y las aletas pélvicas. Las larvas de $B$. houdei con tallas de 1.5 a $1.9 \mathrm{~mm}$ tienen melanóforos en la punta de la mandíbula inferior, en la cabez y en la base de las aletas pectorales. Se observó un mayor desarrollo larvario de B. cantori y B. atlanticus que en especímenes de latitudes mayores.

Palabras clave: bregmacerotidae, Bregmaceros, larvas de peces, desarrollo larvario, Golfo de México.

\section{REFERENCIAS}

Abundio-López, F. 1987. Estudio de la distribución y abundancia larvaria de las familias Bothidae, Soleidae y Cynoglosidae (Pisces: Pleuronectiformes) en el sur del Golfo de México (1983-1984). Tesis de
Licenciatura. Facultad de Ciencias, Universidad Nacional Autonóma de México, México. 50 p.

Blaxter, J.H.S. 1969. Development: Eggs and larvae, p. 177-252. In W.S.Hoar \& D.-J. Randal (eds.). Fish physiology vol. III. Reproduction and growth bioluminescence, pigments and poisons. Academic, Nueva York, EEUU.

Clancey, J.F. 1956. A contribution to the life history of the fish, Bregmaceros atlanticus Goode and Bean, from the Florida Current. Bull. Mar. Sci. Gulf Carib. 6: 233-272.

D'Ancona, U. \& G. Cavinato. 1965. The fishes of the family Bregmacerotidae. Dana Rep. 64: 1-91.

Ditty, J.G. 1989. Separiting early larvae of sciaenids from the western north Atlantic: a review and comparison of larvae off Louisiana and Atlantic coast of the U.S. Bull. Mar. Sci. 44: 1083-1105.

Flores-Coto, C., M. Sánchez-Ramírez \& F. Zavala-García. 1998. Morfología externa del desarrollo larvario de Hemicaranx amblyrhynchus (Pisces: Carangidae) del Sur del Golfo de México. Rev. Biol. Trop. 46: 431-438.

Houde, E.D. 1981. Distribution and abundance of four types of codlet (Pisces: Bregmacerotidae) larvae from the Eastern Gulf of Mexico. Biol. Oceanogr. 1: 81-104.

Houde, E.D. 1984. Bregmacerotidae: development and relationship, p. 300-308. In H.G.Moser, W.-J. Richards, D.-M. Cohen, M.-P. Fahay, A.-W. Kendall, Jr. \& S.-L. Richardson (eds.). Ontogeny and Systematics of Fishes. Am. Soc. Ichthyol. Herpetol. Spec. Publ. 1. La Jolla, California.

Houde, E.D. 1989. Comparative growth, mortality, and energetics of marine fish larvae: temperature and implied latitudinal effects. Fish. Bull. 87: 471-495.

Kendall, Jr. A.W., E.H. Ahlstrom \& H.G. Moser. 1984. Early life history stages of fishes and their characters, p. 11-22. In H.G.Moser, W.-J. Richards, D.-M. Cohen, M.-P. Fahay, A.-W. Kendall, Jr. \& S.-L. Richardson (eds.). Ontogeny and systematics of fishes. Am. Soc. Ichthyol. Herpetol. Spec. Publ. 1. La Jolla, California, EEUU.

Laurence, G.C. 1978. Comparative growth, respiration and delayed feeding abilities of larval cod (Gadus morhua) and haddock (Melanogrammus aeglefinus) as influenced by temperature during laboratory studies. Mar. Biol. 50: 1-7.

Milliken, D.M. \& E.D. Houde. 1984. A new species of Bregmacerotidae (Pisces), Bregmaceros cantori, from the western Atlantic Ocean. Bull. Mar. Sci. 35: 11-19. 
Morse, W.W. 1989. Catchability, growth, and mortality of larval fishes. Fish. Bull. 87: 417-446.

Ordoñez-López, U. \& C. Flores-Coto. 1989. Clave para la identificación de larvas de las familias Gonostomatidae, Sternopthychidae y Myctophidae del sur del Golfo de México. An. Inst. Cienc. del Mar y Limnol. Univ. Nal. Autón. México. 16: 207-222.

Saksena, V.P. \& W.J. Richards. 1986. A new species of gadiform fish, Bregmaceros houdei, from the western North Atlantic. Bull. Mar. Sci. 38: 285-292.

Sánchez-Iturbe, A. 1993. Estudio monográfico de Scyacium gunteri (Pisces: Bothidae) del sur del Golfo de México. Tesis de Maestría. Facultad de Ciencias. Universidad Nacional Autónoma de México. México. 64 p.

Sánchez-Ramírez, M. \& C. Flores-Coto. 1993. Desarrollo larvario y clave de identificación de algunas especies de la familia Carangidae (Pisces) del sur del Golfo de México. An. Inst. Cienc. del Mar y Limnol. Univ. Nal. Autón. México. 20: 1-24.

Shirota, A. 1970. Studies on the mouth size of fish larvae. Bull. of Japan. Soc. of Sci. Fish. 36: 353-368.

Smith, P.E. \& S.L. Richardson. 1979. Técnicas estándar para prospecciones de huevos y larvas de peces pelágicos. FAO Doc. Tec. Pesca. 175: 1-107.

Stevens, E.G. \& H.G. Moser. 1996. Bregmacerotidae: codlets, p. 477-481. In H.G. Moser (ed.). The early stages of fishes in the California current region. CalCOFI Atlas No. 33. La Jolla, California, EEUU.

Zavala-García, F. \& C. Flores-Coto. 1994. Abundancia y distribución de larvas de Bregmacerotidae (Pisces) en la Bahía de Campeche, México. Cienc. Mar. 20: 219-241. 
